\title{
A New Multi-Step Method for Solving Delay Differential Equations using Lagrange Interpolation
}

\author{
V. J. Shaalini ${ }^{a}$, S. E. Fadugba ${ }^{b, *}$ \\ ${ }^{a}$ Department of Mathematics, Bishop Heber College, Trichy, India \\ ${ }^{b}$ Department of Mathematics, Ekiti State University, Ado Ekiti, Nigeria
}

\begin{abstract}
This paper presents 2-step $p$-th order ( $p=2,3,4)$ multi-step methods that are based on the combination of both polynomial and exponential functions for the solution of Delay Differential Equations (DDEs). Furthermore, the delay argument is approximated using the Lagrange interpolation. The local truncation errors and stability polynomials for each order are derived. The Local Grid Search Algorithm (LGSA) is used to determine the stability regions of the method. Moreover, applicability and suitability of the method have been demonstrated by some numerical examples of DDEs with constant delay, time dependent and state dependent delays. The numerical results are compared with the theoretical solution as well as the existing Rational Multi-step Method2 (RMM2).
\end{abstract}

DOI:10.46481/jnsps.2021.247

Keywords: Multi-step method, Delay differential equations, Interpolating function, Lagrange interpolation, Stability polynomial, Stability region

Article History :

Received: 08 June 2021

Received in revised form: 12 July 2021

Accepted for publication: 24 July 2021

Published: 29 August 2021

(C)2021 Journal of the Nigerian Society of Physical Sciences. All rights reserved. Communicated by: T. Latunde

\section{Introduction}

Delay Differential Equations (DDEs) are differential equations in which the derivative of the unknown function depends not only at its present time but also at the previous times. In Ordinary Differential Equations (ODEs), a simple initial condition is given. But to specify DDEs, additional information is needed. Because the derivative depends on the solution at the previous times, an initial history function which gives information about the solution in the past needs to be specified. A general form of the first order DDE is

$$
y^{\prime}(t)=f(t, y(t), y(t-\tau)), \quad t>t_{0}
$$

\footnotetext{
${ }^{*}$ Corresponding author tel. no: +2348067032044

Email address: sunday.f adugba@eksu.edu.ng (S. E. Fadugba )
}

$$
y(t)=\vartheta(t), \quad t \leq t_{0}
$$

where $\vartheta(\mathrm{t})$ is the initial function and $\tau$ is the delay term. The function $\vartheta(\mathrm{t})$ is also known as the 'history function', as it gives information about the solution in the past. If the delay term $\tau$ is a constant, then it is called constant delay. If it is function of time $t$, then it is called time dependent delay. If it is a function of time $t$ and $y(t)$, then it is called state dependent delay.

These equations arise in population dynamics, control systems, chemical kinetic, and in several areas of science and engineering $[1,2,3]$. Recently there has been a growing interest in obtaining the numerical solutions of DDEs. Rostann et al. [4] implemented Adomian decomposition method for the solution of system of DDEs. Two and three point one-step block method for solving DDEs was developed by [5]. Block method for solving Pantograph type functional DDEs was described by 
[6]. An exact/approximate solution of DDEs by using the combination of Laplace and the variational iteration method were obtained by [7]. RK method based on Harmonic Mean for solving DDEs with constant lags was proposed by [8]. Several numerical methods have been constructed for solving stiff DDEs, see $[9,10,11]$. Several multi-step techniques using varieties of interpolating polynomials and functions have been developed to solve ODEs such as $[12,13,14,15,16,17]$, just to mention a few.

In this paper, we present the 2-step p-th order $(p=2,3,4)$ multi-step method for solving DDEs. This method has been referred here as $\operatorname{EPMM}(2, p),(p=2,3,4)$. The organization of this paper is as follows: In Section Two, the derivation of $\operatorname{EPMM}(2, p),(p=2,3,4)$ is given. In Section Three, the stability analysis of $\operatorname{EPMM}(2, p),(\mathrm{p}=2,3,4)$ has been presented. In Section Four, numerical illustrations of DDEs are provided. Moreover, the numerical results are compared with the existing Rational Multi-step Method2 (RMM2) to demonstrate the efficiency and suitability of the method.

\section{Derivation of $\operatorname{EPMM}(2, p)$}

For 2-step p-th order EPMM, let us assume an approximation to the analytical solution $y\left(t_{n+2}\right)$ of (1) given by

$$
y_{n+2}=a_{0} e^{2 h}+1+\sum_{j=1}^{p} b_{j} h^{j}
$$

where $a_{0}, b_{j},(j=1,2, \ldots p)$ are parameters that may contain the approximation of $y\left(t_{n}\right)$ and higher derivatives of $y\left(t_{n}\right)$.

With EPMM in (2) we associate the difference operator $L$ defined by

$$
L[y(t) ; h]_{E P M M}=\left(y(t+2 h)-\left(1+\sum_{j=0}^{p} b_{j} h^{j}\right)\right)-a_{0} e^{2 h}(3)
$$

where $y(t)$ is an arbitrary, continuous and differentiable function.

Expanding $y(t+2 h)$ as Taylor series and collecting terms in (3),

$$
L[y(t) ; h]_{E P M M}=C_{0} h^{0}+C_{1} h^{1}+\cdots+C_{p} h^{p}+C_{p+1} h^{p+1}+. .
$$

where $C_{i}, \quad i=0,1, \ldots, p, p+1$ are the coefficients that need to be determined.

For 2-step second order EPMM, we take $\mathrm{p}=2$ and expand $y(t+$ $2 h$ ) via Taylor series, (3) becomes

$$
\begin{aligned}
& L[y(t) ; h]_{\operatorname{EPMM(2,2)}}=-1+y(t)+h\left(-b_{1}+2 y^{\prime}(t)\right) \\
& +h^{2}\left(2 y^{\prime \prime}(t)-b_{2}\right)+h^{3}\left(\frac{4}{3} y^{\prime \prime \prime}(t)\right)+a_{0} e^{2 h}+O\left(h^{4}\right)
\end{aligned}
$$

Using $e^{2 h} \approx 1+2 h+2 h^{2}$ in (2), we get

$$
\begin{aligned}
& L[y(t) ; h]_{\operatorname{EPMM}(2,2)}=-1-a_{0}+y(t)+h\left(-b_{1}-2 a_{0}+2 y^{\prime}\right. \\
& +h^{2}\left(2 y^{\prime \prime}(t)-2 a_{0}-b_{2}\right)+h^{3}\left(\frac{4}{3} y^{\prime \prime \prime}(t)\right)+a_{0} e^{2 h}+O\left(h^{4}\right)(6)
\end{aligned}
$$

We consider a commonly used linear test equation with a constant delay $\tau=m h$ where $\mathrm{m}$ is a positive integer,

$$
y^{\prime}(t)=\lambda y(t)+\mu y(t-\tau), t>t_{0}
$$

For second order EPMM, we put $C_{0}=C_{1}=C_{2}=0$ in (7) and get the following solutions:

$$
a_{0}=y(t)-1, b_{1}=2\left(y^{\prime}(t)-y(t)+1\right), b_{2}=2\left(y^{\prime \prime}(t)-y(t)+1\right)(8)
$$

If we write $y_{n}=y\left(t_{n}\right)$ and $y_{n}^{(m)}=y^{(m)}\left(t_{n}\right)$ for $\mathrm{m}=1,2, \ldots$, then

(8) becomes

$$
\left\{\begin{array}{l}
A=y_{n} \\
b_{1}=2\left(y_{n}^{\prime}-y_{n}+1\right) \\
b_{2}=2\left(y_{n}^{\prime \prime}-y_{n}+1\right)
\end{array}\right.
$$

Taking $p=2$ and $e^{2 h} \approx 1+2 h+2 h^{2}$ in (2), we get

$$
y_{n+2}=\left(a_{0}+1\right)+h\left(2 a_{0}+b_{1}\right)+h^{2}\left(2 a_{0}+b_{2}\right)
$$

Substituting (9) into (10), we have

$$
y_{n+2}=y_{n}+2 h y_{n}{ }^{\prime}+2 h^{2} y_{n}{ }^{\prime \prime}
$$

The local truncation error of EPMM $(2,2)$ is given by,

$$
\operatorname{LTE} E_{\operatorname{EPMM(2,2)}}=h^{3}\left(\frac{4}{3} y_{n}^{\prime \prime \prime}\right)+O\left(h^{4}\right)
$$

Taking $\mathrm{p}=3$ in (2) and on simplification, we get the formula for $\operatorname{EPMM}(2,3)$

$$
y_{n+2}=y_{n}+2 h y_{n}^{\prime}+2 h^{2} y_{n}^{\prime \prime}+\frac{4}{3} h^{3} y_{n}^{\prime \prime \prime}
$$

The local truncation error of $\operatorname{EPMM}(2,3)$ is given by,

$$
\operatorname{LTE} E_{\operatorname{EPMM}(2,3)}=h^{4}\left(\frac{2}{3} y_{n}{ }^{(4)}\right)+O\left(h^{5}\right)
$$

Taking $\mathrm{p}=4$ in (2) and on simplification, we get the formula for $\operatorname{EPMM}(2,4)$

$$
y_{n+2}=y(t)+2 h y^{\prime}(t)+2 h^{2} y^{\prime \prime}(t)+\frac{4}{3} h^{3} y^{\prime \prime \prime}(t)+\frac{2}{3} h^{4} y^{(4)}(t)(13)
$$

The local truncation error of $\operatorname{EPMM}(2,4)$ is given by,

$$
\operatorname{LTE} E_{E P M M(2,4)}=h^{5}\left(\frac{4}{15} y_{n}^{(5)}\right)+O\left(h^{6}\right)
$$

\section{Stability Analysis of EPMM}

In this section, we derive the stability polynomials of EPMM $(2, p),(\mathrm{p}=2,3,4)$ and their corresponding stability regions 
$y(t)=\phi(t), t \leq t_{0}$

where $\lambda, \mu \in C, \tau>0$ and $\Phi$ is continuous.

Using (11) in (14), we get

$$
\begin{aligned}
& y_{n+2}=y_{n}+2 h\left(\lambda y_{n}+\mu y\left(t_{n}-\tau\right)\right)+2 h^{2}\left(\lambda y_{n}^{\prime}+\mu y^{\prime}\left(t_{n}-\tau\right)\right)( \\
& y\left(t_{n}-m h\right)=y\left(t_{n-m}\right)=\sum_{l=-r_{1}}^{s_{1}} L_{l}\left(c_{i}\right) y_{n-m+l}
\end{aligned}
$$

with

$$
L_{l}\left(c_{i}\right)=\prod_{j=-r_{1}}^{s_{1}} \frac{c_{i}-j_{1}}{l-j_{1}}, \quad j_{1} \neq l \quad \text { and } r_{1}, s_{1}>0
$$

Taking

$$
y\left(t_{n}-\tau\right)=\sum_{l=-r_{1}}^{s_{1}} L_{l}(c) y_{n-m+l}
$$

and

$$
y^{\prime}\left(t_{n}-\tau\right)=\lambda \sum_{l=-r_{1}}^{s_{1}} L_{l}(c) y_{n-m+l}+\mu \sum_{l=-r_{1}}^{s_{1}} L_{l}(c) y_{n-2 m+l}
$$

Then (15) becomes

$$
\begin{aligned}
& y_{n+2}=y_{n}+2 h\left(\lambda y_{n}+\mu \sum_{l=-r_{1}}^{s_{1}} L_{l}(c) y_{n-m+l}\right) \\
& +2 h^{2}\left(\begin{array}{c}
\lambda\left(\lambda y_{n}+\mu \sum_{l=-r_{1}}^{s_{1}} L_{l}(c) y_{n-m+l}\right) \\
+\mu \lambda \sum_{l=-r_{1}}^{s_{1}} L_{l}(c) y_{n-m+l} \\
+\mu \sum_{l=-r_{1}}^{s_{1}} L_{l}(c) y_{n-2 m+l}
\end{array}\right) \\
& y_{n+2}=y_{n}+2 \lambda h y_{n}+2 \lambda^{2} h^{2} y_{n} \\
& +\sum_{l=-r_{1}}^{s_{1}} L_{l}(c) y_{n-m+l}\left(2 \mu h+4 h^{2} \mu \lambda\right)+\sum_{l=-r_{1}}^{s_{1}} L_{l}(c) y_{n-2 m+l}\left(2 h^{2} \mu^{2}\right) \\
& \quad y_{n+2}=y_{n}\left(1+2 \lambda h+2(\lambda h)^{2}\right)+\sum_{l=-r_{1}}^{s_{1}} L_{l}(c) y_{n-m+l}(\mu h(2+4 \lambda h))^{2} \\
& +\sum_{l=-r_{1}}^{s_{1}} L_{l}(c) y_{n-2 m+l}\left(2(\mu h)^{2}\right) \\
& \text { Let } \alpha=\lambda h \text { and } \beta=\mu h \text { then the above equation becomes } \\
& +2 \beta^{2} \sum_{l=-r_{1}}^{s_{1}} L_{l}(c) y_{n-2 m+l} \\
& y_{n+2}=y_{n}\left(1+2 \alpha+2 \alpha^{2}\right)+(\beta(2+4 \alpha)) \sum_{l=-r_{1}}^{s_{1}} L_{l}(c) y_{n-m+l}
\end{aligned}
$$

To obtain the stability polynomial, the delay term is approximated using three points Lagrange interpolation.

By putting $n-m+l=0$ and $n-2 m+l=0$ and by taking $l=$ $-1,0,1$, the stability polynomial will be in the standard form.
The recurrence is stable if the zeros of $\zeta_{i}$ of the stability polynomial

$$
\begin{aligned}
& S(\alpha, \beta: \zeta)=\zeta^{n+2}-\left(1+\alpha+2 \alpha^{2}\right) \zeta^{n} \\
& -\beta(2+4 \alpha)\left(L_{-1}(c)+L_{0}(c) \zeta+L_{1}(c) \zeta^{2}\right) \\
& -2 \beta^{2}\left(L_{-1}(c)+L_{0}(c) \zeta+L_{1}(c) \zeta^{2}\right)
\end{aligned}
$$

satisfies the root condition $\left|\zeta_{i}\right| \leq 1$. From this, the stability polynomial for the method $\operatorname{GRMM}(2,2)$ with $\tau=1$ is given as

$$
S(\alpha, \beta: \zeta)=\zeta^{n+2}-\left(1+\alpha+2 \alpha^{2}\right) \zeta^{n}-\left(2 \beta+2 \beta^{2}+4 \alpha \beta\right)
$$

Similarly, by considering suitable number of points in Lagrange interpolation according to the order of the method, we can obtain the corresponding stability polynomials of $\operatorname{EPMM}(2, p)$. When $p=3$, the stability polynomial for $\operatorname{EPMM}(2,3)$ is given as

$$
\begin{aligned}
& S(\alpha, \beta: \zeta)=\zeta^{n+2}-\left(1+\alpha+2 \alpha^{2}+\frac{4}{3} \alpha^{3}\right) \zeta^{n} \\
& -\left(2 \beta+2 \beta^{2}+\frac{4}{3} \beta^{3}+4 \alpha \beta+4 \alpha^{2} \beta+4 \alpha \beta^{2}\right)
\end{aligned}
$$

When $p=4$, the stability polynomial for $\operatorname{EPMM}(2,4)$ is given as

$$
\begin{array}{r}
S(\alpha, \beta: \zeta)=\zeta^{n+2}-\left(1+\alpha+2 \alpha^{2}+\frac{4}{3} \alpha^{3}+\frac{2}{3} \alpha^{4}\right) \zeta^{n} \\
-\left(2 \beta+2 \beta^{2}+\frac{4}{3} \beta^{3}+\frac{2}{3} \beta^{4}+2 \alpha \beta+4 \alpha^{2} \beta+4 \alpha \beta^{2}+\frac{8}{3} \alpha \beta^{3}\right. \\
\left.+\frac{8}{3} \alpha^{3} \beta+4 \alpha^{2} \beta^{2}\right)
\end{array}
$$

The stability regions of $\operatorname{EPMM}(2,2), \operatorname{EPMM}(2,3)$ and $\operatorname{EPMM}(2,4)$ are given in Figures $1-3$.

In a similar manner, we can obtain the stability polynomials and their corresponding regions of EPMM with $r$-step and of any order $p$.

\section{Numerical Examples}

Example 1: (Stiff linear system with multiple delays)

$$
\begin{aligned}
& y_{1}^{\prime}(t)=-\frac{1}{2} y_{1}(t)-\frac{1}{2} y_{2}(t-1)+f_{1}(t), \\
& y_{2}^{\prime}(t)=-y_{2}(t)-\frac{1}{2} y_{1}\left(t-\frac{1}{2}\right)+f_{2}(t), 0 \leq t \leq 1
\end{aligned}
$$

with initial conditions

$$
\begin{aligned}
& y_{1}(t)=e^{-t / 2}, \frac{-1}{2} \leq t \leq 0, \\
& y_{2}(t)=e^{-t},-1 \leq t \leq 0
\end{aligned}
$$


Table 1. Comparison of Absolute Error Results in EPMM and RMM2 for Example 1

\begin{tabular}{cccccccc}
\hline Time $(\mathrm{t})$ & $\mathrm{Y}$ & EPMM $(2,2)$ & RMM2 $(2,2)$ & EPMM $(2,3)$ & RMM2 $(2,3)$ & EPMM $(2,4)$ & RMM2(2, 4) \\
\hline 0.2 & $\mathrm{y}_{1}$ & $1.52 \mathrm{e}-06$ & $7.54 \mathrm{e}-07$ & $3.80 \mathrm{e}-09$ & $1.26 \mathrm{e}-09$ & $7.60 \mathrm{e}-12$ & $9.06 \mathrm{e}-07$ \\
\hline & $\mathrm{y}_{2}$ & $3.26 \mathrm{e}-04$ & $3.31 \mathrm{e}-04$ & $3.26 \mathrm{e}-04$ & $3.26 \mathrm{e}-04$ & $3.15 \mathrm{e}-04$ & $3.32 \mathrm{e}-04$ \\
\hline 0.4 & $\mathrm{y}_{1}$ & $2.75 \mathrm{e}-06$ & $1.36 \mathrm{e}-06$ & $6.88 \mathrm{e}-09$ & $2.28 \mathrm{e}-09$ & $1.38 \mathrm{e}-11$ & $1.64 \mathrm{e}-06$ \\
\hline & $\mathrm{y}_{2}$ & $5.62 \mathrm{e}-04$ & $5.71 \mathrm{e}-04$ & $5.62 \mathrm{e}-04$ & $5.62 \mathrm{e}-04$ & $5.43 \mathrm{e}-04$ & $5.72 \mathrm{e}-04$ \\
\hline 0.6 & $\mathrm{y}_{1}$ & $3.73 \mathrm{e}-06$ & $1.85 \mathrm{e}-06$ & $9.33 \mathrm{e}-09$ & $3.10 \mathrm{e}-09$ & $1.87 \mathrm{e}-11$ & $2.22 \mathrm{e}-06$ \\
\hline & $\mathrm{y}_{2}$ & $7.27 \mathrm{e}-04$ & $7.38 \mathrm{e}-04$ & $7.27 \mathrm{e}-04$ & $7.27 \mathrm{e}-04$ & $7.04 \mathrm{e}-04$ & $7.40 \mathrm{e}-04$ \\
\hline 0.8 & $\mathrm{y}_{1}$ & $4.50 \mathrm{e}-06$ & $2.23 \mathrm{e}-06$ & $1.13 \mathrm{e}-08$ & $3.73 \mathrm{e}-09$ & $2.25 \mathrm{e}-11$ & $2.68 \mathrm{e}-06$ \\
\hline & $\mathrm{y}_{2}$ & $8.36 \mathrm{e}-04$ & $8.48 \mathrm{e}-04$ & $8.36 \mathrm{e}-04$ & $8.36 \mathrm{e}-04$ & $8.12 \mathrm{e}-04$ & $8.51 \mathrm{e}-04$ \\
\hline 1.0 & $\mathrm{y}_{1}$ & $5.09 \mathrm{e}-06$ & $2.53 \mathrm{e}-06$ & $1.27 \mathrm{e}-08$ & $4.22 \mathrm{e}-09$ & $2.55 \mathrm{e}-11$ & $3.04 \mathrm{e}-06$ \\
\hline & $\mathrm{y}_{2}$ & $9.03 \mathrm{e}-04$ & $9.16 \mathrm{e}-04$ & $9.03 \mathrm{e}-04$ & $9.03 \mathrm{e}-04$ & $8.78 \mathrm{e}-04$ & $9.18 \mathrm{e}-04$ \\
\hline
\end{tabular}

Table 2. Comparison of Absolute Error Results in EPMM and RMM2 for Example 2

\begin{tabular}{ccccccc}
\hline Time $(\mathrm{t})$ & EPMM $(2,2)$ & RMM2 $(2,2)$ & EPMM $(2,3)$ & RMM2 $(2,3)$ & EPMM $(2,4)$ & RMM2 $(2,4)$ \\
\hline 1.1 & $1.82 \mathrm{e}-06$ & $4.04 \mathrm{e}-06$ & $1.31 \mathrm{e}-06$ & $1.46 \mathrm{e}-06$ & $1.35 \mathrm{e}-06$ & $9.58 \mathrm{e}-06$ \\
1.2 & $9.26 \mathrm{e}-07$ & $2.51 \mathrm{e}-06$ & $1.25 \mathrm{e}-06$ & $1.20 \mathrm{e}-06$ & $2.75 \mathrm{e}-06$ & $8.03 \mathrm{e}-06$ \\
1.3 & $1.50 \mathrm{e}-06$ & $3.23 \mathrm{e}-08$ & $2.22 \mathrm{e}-06$ & $4.13 \mathrm{e}-06$ & $2.26 \mathrm{e}-07$ & $8.41 \mathrm{e}-06$ \\
1.4 & $3.50 \mathrm{e}-06$ & $5.92 \mathrm{e}-06$ & $2.70 \mathrm{e}-06$ & $3.29 \mathrm{e}-06$ & $2.99 \mathrm{e}-06$ & $1.19 \mathrm{e}-05$ \\
1.5 & $4.95 \mathrm{e}-05$ & $1.96 \mathrm{e}-06$ & $3.13 \mathrm{e}-06$ & $6.16 \mathrm{e}-06$ & $4.39 \mathrm{e}-06$ & $6.83 \mathrm{e}-06$ \\
\hline
\end{tabular}

Table 3. Comparison of Absolute Error Results in EPMM and RMM2 for Example 3

\begin{tabular}{ccccccc}
\hline Time $(\mathrm{t})$ & EPMM $(2,2)$ & RMM2 $(2,2)$ & EPMM $(2,3)$ & RMM2 $(2,3)$ & EPMM $(2,4)$ & RMM2 $(2,4)$ \\
\hline 0.2 & $1.33 \mathrm{e}-05$ & $1.35 \mathrm{e}-05$ & $1.97 \mathrm{e}-09$ & $2.36 \mathrm{e}-07$ & $4.92 \mathrm{e}-09$ & $4.87 \mathrm{e}-07$ \\
0.4 & $2.60 \mathrm{e}-05$ & $2.80 \mathrm{e}-05$ & $2.46 \mathrm{e}-08$ & $5.62 \mathrm{e}-07$ & $1.76 \mathrm{e}-09$ & $5.46 \mathrm{e}-06$ \\
0.6 & $3.78 \mathrm{e}-05$ & $4.48 \mathrm{e}-05$ & $5.67 \mathrm{e}-08$ & $7.45 \mathrm{e}-07$ & $3.03 \mathrm{e}-09$ & $3.58 \mathrm{e}-05$ \\
0.8 & $4.79 \mathrm{e}-05$ & $6.56 \mathrm{e}-05$ & $9.93 \mathrm{e}-08$ & $8.72 \mathrm{e}-07$ & $2.45 \mathrm{e}-09$ & $2.32 \mathrm{e}-04$ \\
1.0 & $5.63 \mathrm{e}-05$ & $9.31 \mathrm{e}-05$ & $2.85 \mathrm{e}-06$ & $2.85 \mathrm{e}-06$ & $3.24 \mathrm{e}-09$ & $3.24 \mathrm{e}-04$ \\
\hline
\end{tabular}

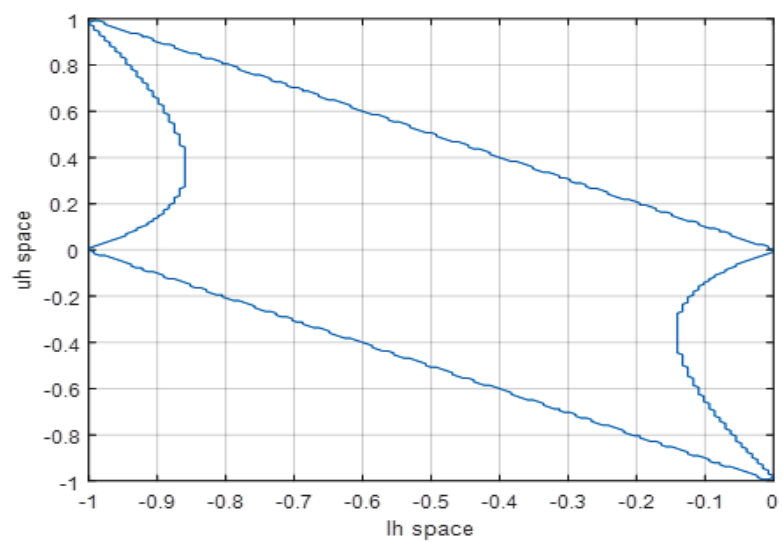

Figure 1. Stability Region of 2-step Second Order EPMM

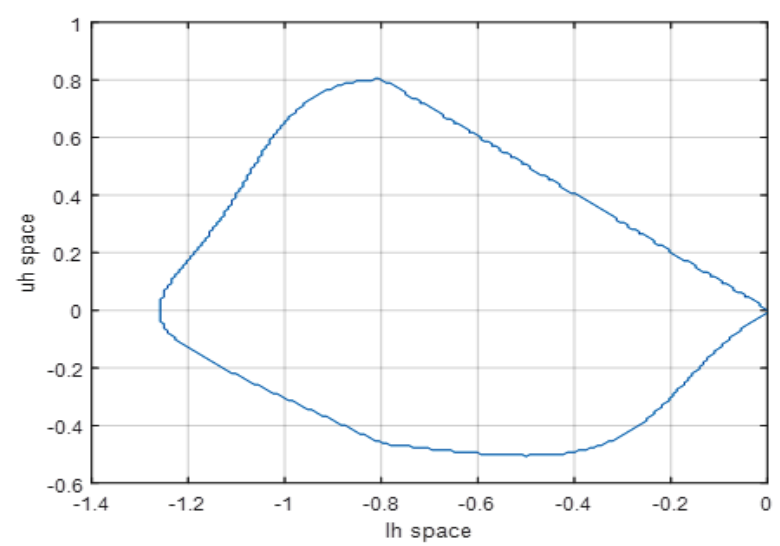

Figure 2. Stability Region of 2-step Third Order EPMM

where

$$
f_{1}(t)=\frac{1}{2} e^{-(t-1)}
$$

and

$$
f_{2}(t)=\frac{1}{2} e^{-(t-1 / 2) / 2}
$$

The exact solution is given by

$$
y_{1}(t)=e^{-t / 2}, y_{2}(t)=e^{-t}
$$

Example 2: (Time-dependent delay)

$$
y^{\prime}(t)=\frac{t-1}{t} y(\ln (t)-1) y(t), 1 \leq t \leq \frac{3}{2}
$$

With initial condition

$$
y(t)=1,0 \leq t \leq 1
$$




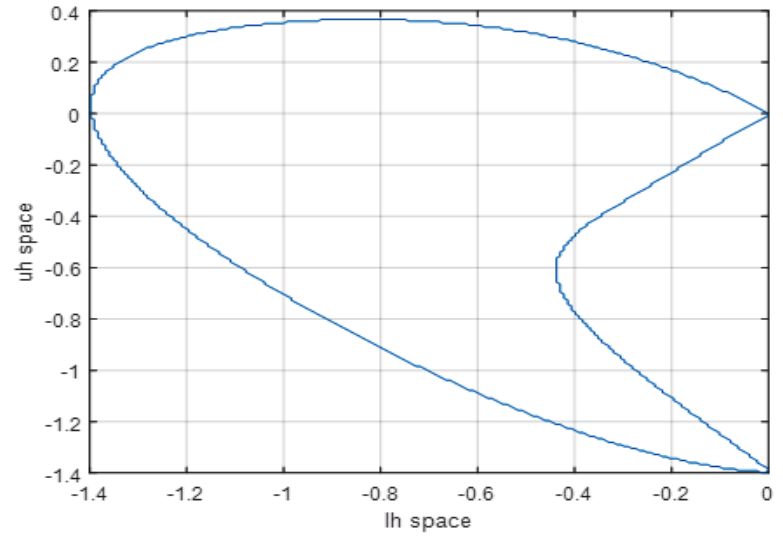

Figure 3. Stability Region of 2-step Fourth Order EPMM

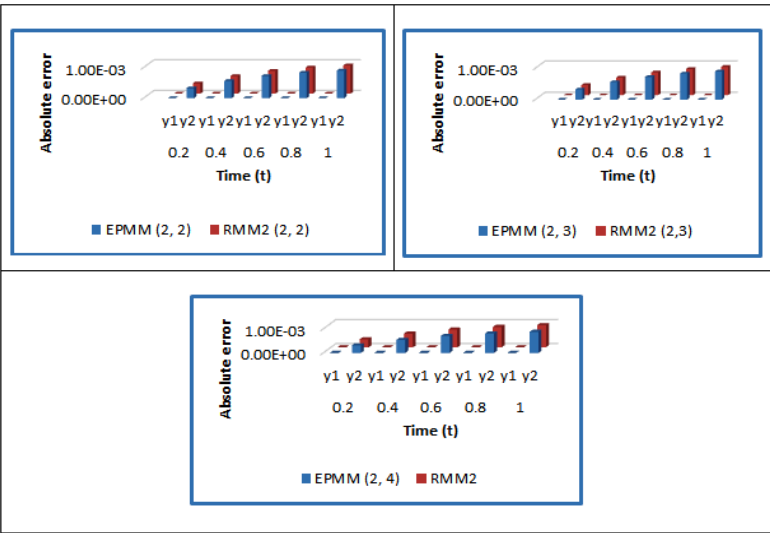

Figure 4. Comparison of Error Graph of $y_{1}$ and $y_{2}$ in Example 1

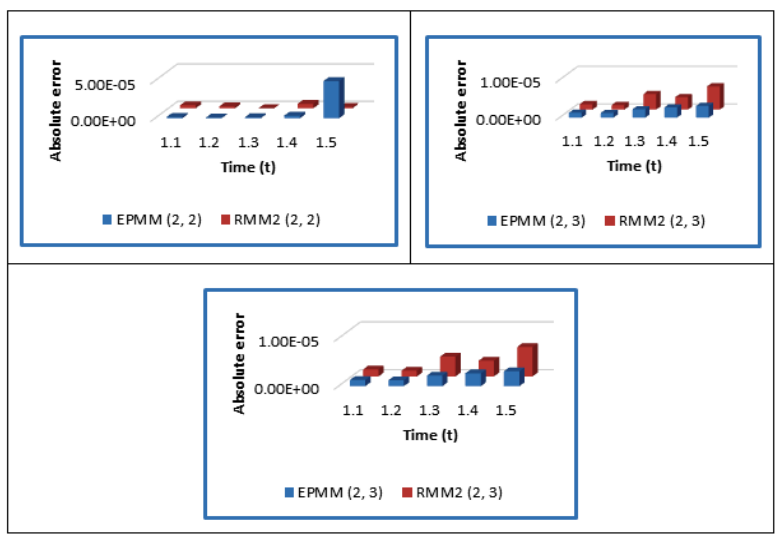

Figure 5. Comparison of Absolute Error Graph of $y$ in Example 2

and the exact solution is given by

$$
y(t)=\exp (t-\ln (t)-1), 1 \leq t \leq \frac{3}{2}
$$

\section{Example 3: (State-dependent delay)}

$$
y^{\prime}(t)=\cos (t) y(\mathrm{y}(\mathrm{t})-2), t \geq 0
$$

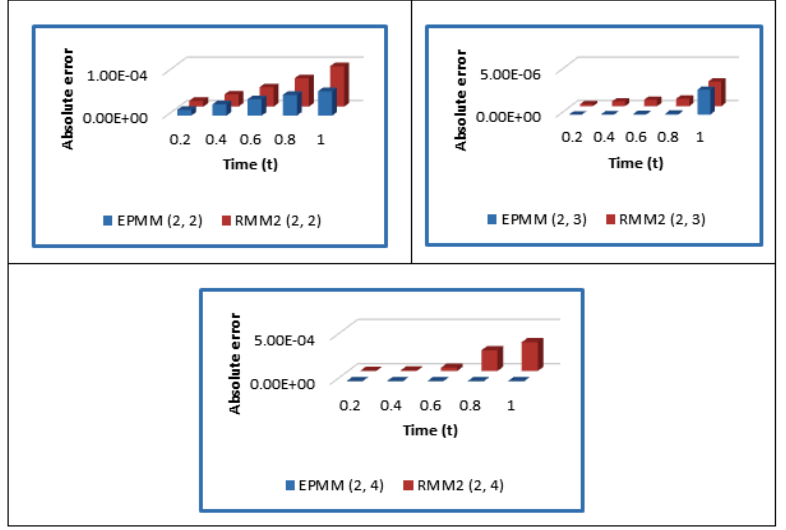

Figure 6. Comparison of Absolute Error Graph of $y$ in Example 3

With initial condition,

$$
y(t)=1, t \leq 0
$$

and the exact solution is given by

$$
y(t)=\sin (t)+1,0 \leq t \leq 1
$$

By taking the step-size $\mathrm{h}=0.01$ in the above examples, the absolute errors by using EPMM and RMM 2 are given in Tables $1-3$ and their corresponding error graphs are shown in Figures $4-6$.

\section{Conclusion}

In this paper, the new multi-step method of r-step and p-th order that are based on interpolating functions which consists of both polynomial and exponential function is presented for solving DDEs. The local truncation errors have been determined. The stability polynomials of $\operatorname{EPMM}(2, p)$ where $p=2,3,4$ are derived and their corresponding stability regions are obtained and shown in Figures 1-3. The delay argument is approximated using Lagrange interpolation. Numerical examples of DDEs with constant delay, time dependent delay and state dependent delays have been considered to demonstrate the efficiency of the proposed method. The comparative absolute error analyses of EPMM (2,p) in the context of RMM2 (2,p) for Examples 1, 2 and 3 were shown in Tables 1, 2 and 3, respectively. From the Figures $4-6$, it is evident that the newly proposed method gives results with good accuracy than the existing RMM2. Hence, it is concluded that the proposed $\operatorname{EPMM}(2, p)$ is suitable for solving DDEs.

\section{Acknowledgments}

We thank the referees and the editor for their contributions on the improvement of this paper. 


\section{References}

[1] Y. Kuang, Delay differential equations with applications in population dynamics, Academic Press, Boston, San Diego, New York. (1993).

[2] E. Fridman, L. Fridman \& E. Shusti, "Steady modes in relay control systems with time delay and periodic disturbances", Journal of Dynamical Systems Measurement and Control 122 (2000) 732.

[3] I. Epstein \& Y. Luo, "Differential delay equations in chemical kinetics: non-linear models; the cross-shaped phase diagram and the oregonator", Journal of Chemical Physic 95 (1991) 244.

[4] R. K. Saeed \& B. M. Rahman, "Adomian decomposition method for solving system of delay differential equation", Australian Journal of Basic and Applied Sciences 4 (2010) 3613.

[5] H. M. Radzi, Z. A. Majid, F. Ismail \& M. Suleiman, "Two and three point one-step block method for solving delay differential equations", Journal of Quality Measurement and Analysis 8 (2012) 29.

[6] F. Ishak, M. B. Suleiman \& Zanariah A. Majid, "Block Method for solving Pantograph-type functional differential equations", Proceeding of the World Congress on Engineering 2 (2013).

[7] T. A. Biala, O. O. Asim \& Y. O. Afolabi, "A combination of the Laplace and the variational iteration method for the analytical treatment of delay differential equations", International Journal of Differential Equations and Applications 13 (2014) 164.

[8] A. E. K. Puhpam \& J. V. Shaalini, "Solving delay differential equations with constant lags using RKHaM method", International Journal of Scientific Research 5 (2016) 585.

[9] G. A. Bocharov, G. I. Marchuk \& A .A. Romanyukha, "Numerical so- lution by LMMs of stiff delay differential systems modelling an immune response", Numerische Mathematik 73 (1996) 131.

[10] J. Vinci Shaalini \& A. Emimal Kanaga Pushpam, "Analysis of Composite Runge Kutta Methods and New One-step Technique for stiff Delay Differential Equations", IAENG International Journal of Applied Mathematics 4 (2019) 359.

[11] J. V. Shaalini \& A. E. K. Pushpam, "A new one step method for solving stiff and non-stiff delay differential equations using Lagrange interpolation", Journal of Applied Science and Computations 6 (2019) 949.

[12] S. O. Fatunla, "Nonlinear multistep methods for initial value problems", Computers and Mathematics with Applications 8 (1982) 231.

[13] K. O. Okosun \& R. A. Ademiluyi, "A two-step second order inverse polynomial methods for integration of differential equations with singularities", Research Journal of Applied Sciences 2 (2007) 13.

[14] O. E. Abolarin \& S. W. Akingbade, "Derivation and application of fourth stage inverse polynomial scheme to initial value problems", IAENG International Journal of Applied Mathematics 47 (2017) 459.

[15] T. Y. Ying \& N. Yaacob, "A new class of rational multi-step methods for solving initial value problems", Malaysian Journal of Mathematical Sciences, 7 (2013) 31

[16] J. Vinci Shaalini, A. \& E. K. Pushpam, "A Class of 2-Step fourth order rational multi-step methods for solving delay differential equations", International Journal for Research in Engineering Application \& Management 5 (2019) 164.

[17] S. E. Fadugba, S.N. Ogunyebi \& B.O. Falodun, "An examination of a second order numerical method for solving initial value problems", Journal of the Nigerian Society of Physical Sciences 2 (2020) 120 